\title{
Nonstandard Analysis By Means of IDEAL VALUES OF SEQUENCES
}

\author{
Mauro Di Nasso
}

\begin{abstract}
A notion of ideal value of $N$-sequences is axiomatized through elementary properties. The resulting theory ZFC $[\boldsymbol{\alpha}]$ provides nonstandard analysis with a general foundational framework.
\end{abstract}

1991 MSC. Primary: 26E35, 03E70, 03H05. Secondary: 03C20, 03E35.

In this paper, the axiomatic system $\mathrm{ZFC}[\boldsymbol{\alpha}]$ is presented. It is a generalization in a set theoretic context of an approach to nonstandard analysis recently given by $\mathrm{V}$. Benci in $[\mathrm{BE}]$. In that paper, nonstandard analysis is presented by postulating, besides a nonstandard embedding $*: V(X) \rightarrow V(X)$ from a superstructure into itself, the existence of a pre-extension for each function defined on the set $N$ of natural numbers. Axioms are then given that correlate pre-extensions with the enlarging map $*$. Here, we formalize the idea of preextension by means of a notion of ideal value. Precisely, we enrich the language of set theory with a constant symbol $\boldsymbol{\alpha}$ (to be intended as a new ideal natural number) and axiomatize the ideal value $\varphi(\boldsymbol{\alpha})$ of $N$-sequences $\varphi$.

The idea of adding a new logical constant to behave as an "infinite" natural number is not new. Precisely, it goes back to the pioneering work by $\mathrm{C}$. Schmieden and D. Laugwitz [SL], appeared before Robinson's presentation of nonstandard analysis. A general way of formally giving a "nonstandard extension" of a given first order theory by means of a new constant symbol, can be found in Laugwitz' work [LA].

In this paper, an approach is given in the full generality of set theory. As a consequence of our axioms, a nonstandard embedding $*: \mathbf{V} \rightarrow \mathbf{V}$ is determined for the universal class of all sets, and all basic principles of nonstandard analysis, including transfer and countable saturation, are proved. All principles of mathematics as formalized by Zermelo-Fraenkel set theory with choice are assumed, with the only exception of regularity. Thus, working in ZFC $[\boldsymbol{\alpha}]$, one can manipulate (external) sets as in ordinary mathematics with no restrictions. Also, nonstandard arguments can be iterated, because the enlarging map $*$ is defined for all sets. For instance, one can consider the hyper-hyperreal numbers 
${ }^{*}\left({ }^{*} R\right)$ or the nonstandard extension ${ }^{*} \mathcal{L}$ of a Loeb measure space $\mathcal{L}$ etc. Another feature of our axiomatic system ZFC $[\boldsymbol{\alpha}]$ is that it consists of few simple properties expressed in elementary terms, thus making it possible to present them even at the freshman level. ${ }^{1}$ For basic notions from set theory and model theory, we refer to $[\mathrm{JE}]$ and $[\mathrm{CK}]$, respectively.

Let $L=\{\in, \boldsymbol{\alpha}\}$ be the language of set theory together with a new constant symbol $\boldsymbol{\alpha}$. ZFC $[\boldsymbol{\alpha}]$ is the theory of $L$ given by the following seven axioms. ${ }^{2}$

1. $Z F C^{-}$

All principles of mathematics, as axiomatized by Zermelo-Fraenkel set theory with choice but without regularity, are assumed. The replacement and separation schemata are also considered for formulas where the symbol $\boldsymbol{\alpha}$ occurs. ${ }^{3}$

2. $\boldsymbol{\alpha} \notin N$.

3. Every sequence $\varphi: N \rightarrow A$ has a unique ideal value $\varphi(\boldsymbol{\alpha}){ }^{4}$

For simplicity, we will use the same symbol to denote a sequence $\varphi: N \rightarrow A$ and its pre-extension $\varphi: N \cup\{\boldsymbol{\alpha}\} \rightarrow A \cup\{\varphi(\boldsymbol{\alpha})\}$. The remaining axioms give some natural properties of the ideal values.

4. The ideal extension of the identity map $1_{N}: n \mapsto n$ on $N$ is the identity map on $N \cup\{\boldsymbol{\alpha}\}$, i.e. $1_{N}(\boldsymbol{\alpha})=\boldsymbol{\alpha}$.

5. For every sequence $\varphi$ :

$$
\varphi(\boldsymbol{\alpha})=\{\psi(\boldsymbol{\alpha}): \psi \text { is a sequence with } \psi(n) \in \varphi(n) \text { for all } n \in N\}
$$

In other words, the elements of the ideal value $\varphi(\boldsymbol{\alpha})$ are precisely the ideal values of sequences that are "point-wise" elements of $\varphi$. In particular, if $c_{\emptyset}$ is the sequence constantly equal to the emptyset, then $c_{\emptyset}(\boldsymbol{\alpha})=\emptyset$. The next axiom states that pairs are preserved at the ideal point $\boldsymbol{\alpha}$.

6. If $\vartheta(n)=\{\varphi(n), \psi(n)\}$ for all $n \in N$, then $\vartheta(\boldsymbol{\alpha})=\{\varphi(\boldsymbol{\alpha}), \psi(\boldsymbol{\alpha})\}$.

The last axiom gives a natural coherence property of ideal values with respect to compositions.

7. If $\varphi, \psi$ are sequences taking values in a set $E$, and if $f$ is any function defined on $E$, then

$$
\varphi(\boldsymbol{\alpha})=\psi(\boldsymbol{\alpha}) \Rightarrow(f \circ \varphi)(\boldsymbol{\alpha})=(f \circ \psi)(\boldsymbol{\alpha})
$$

\footnotetext{
${ }^{1}$ A more general nonstandard set theory where unlimited levels of saturation are available, is presented in [BD].

${ }^{2}$ In the following, by sequence we will always mean a function whose domain is the set $N$ of natural numbers.

${ }^{3}$ Actually, this is not an axiom but an axiom scheme.

${ }^{4}$ We remark that in general $\varphi(\boldsymbol{\alpha}) \notin A$.
} 
Quite surprisingly, we will see that the above axioms are all that is needed in order to get a countably saturated nonstandard embedding $*$ defined for all sets. We remark that in the above presentation, not a single technical notion from mathematical logic is used. In fact, axiom 1 can be skipped by implicitly assuming all "usual" principles of mathematics; and the remaining axioms are all expressed in truly basic terms, such as pairs or compositions of functions. Let us start developing our theory. Axiom 6 says that pairs are preserved at ideal values. As a first immediate result, we see next that ideal values also preserve inclusions, ordered pairs, and other basic set operations.

Proposition 1. Let $\varphi, \psi, \vartheta$ be any $N$-sequences. Then

(i) $\varphi(n) \subseteq \psi(n)$ for all $n \in N \Rightarrow \varphi(\boldsymbol{\alpha}) \subseteq \psi(\boldsymbol{\alpha})$.

(ii) $\varphi(n)=\bigcup \psi(n)$ for all $n \in N \Rightarrow \varphi(\boldsymbol{\alpha})=\bigcup \psi(\boldsymbol{\alpha}){ }^{5}$

(iii) $\vartheta(n)=\varphi(n) \cup \psi(n)$ for all $n \in N \Rightarrow \vartheta(\boldsymbol{\alpha})=\varphi(\boldsymbol{\alpha}) \cup \psi(\boldsymbol{\alpha})$.

(iv) $\vartheta(n)=\varphi(n) \cap \psi(n)$ for all $n \in N \Rightarrow \vartheta(\boldsymbol{\alpha})=\varphi(\boldsymbol{\alpha}) \cap \psi(\boldsymbol{\alpha})$.

(v) $\vartheta(n)=\varphi(n) \backslash \psi(n)$ for all $n \in N \Rightarrow \vartheta(\boldsymbol{\alpha})=\varphi(\boldsymbol{\alpha}) \backslash \psi(\boldsymbol{\alpha})$.

(vi) $\vartheta(n)=\langle\varphi(n), \psi(n)\rangle$ for all $n \in N \Rightarrow \vartheta(\boldsymbol{\alpha})=\langle\varphi(\boldsymbol{\alpha}), \psi(\boldsymbol{\alpha})\rangle$.

(vii) $\vartheta(n)=\varphi(n) \times \psi(n)$ for all $n \in N \Rightarrow \vartheta(\boldsymbol{\alpha})=\varphi(\boldsymbol{\alpha}) \times \psi(\boldsymbol{\alpha})$.

Proof. All proofs are straightforward applications of the axioms, thus here we only give two of them as examples. (iii) Notice that $\vartheta(n)=\varphi(n) \cup \psi(n) \Leftrightarrow$ $\vartheta(n)=\bigcup \xi(n)$ where $\xi(n)=\{\varphi(n), \psi(n)\}$. By (ii) $\vartheta(\boldsymbol{\alpha})=\bigcup \xi(\boldsymbol{\alpha})$, and by axiom $6 \xi(\boldsymbol{\alpha})=\{\varphi(\boldsymbol{\alpha}), \psi(\boldsymbol{\alpha})\}$. Thus $\vartheta(\boldsymbol{\alpha})=\bigcup\{\varphi(\boldsymbol{\alpha}), \psi(\boldsymbol{\alpha})\}$, the thesis. (v) Notice that $\vartheta(n)=\varphi(n) \backslash \psi(n) \Leftrightarrow \vartheta(n) \cap \psi(n)=c_{\emptyset}(n)$ and $\vartheta(n) \cup(\varphi(n) \cap$ $\psi(n))=\varphi(n)$. By considering the ideal values and properties (iii) and (iv), one gets $\vartheta(\boldsymbol{\alpha}) \cap \psi(\boldsymbol{\alpha})=\emptyset$ and $\vartheta(\boldsymbol{\alpha}) \cup(\varphi(\boldsymbol{\alpha}) \cap \psi(\boldsymbol{\alpha}))=\varphi(\boldsymbol{\alpha})$. We conclude that $\vartheta(\boldsymbol{\alpha})=\varphi(\boldsymbol{\alpha}) \backslash \psi(\boldsymbol{\alpha})$.

It is straightforwardly seen by induction that the above properties also hold for finite unions, finite intersections, finite (ordered) tuples and finite cartesian products. Axiom 5 implies that the sequence constantly equal to the emptyset is stable at the ideal value. The next result extends that property to every (von Neumann) natural number.

Proposition 2. Let $n$ be any given natural number and let $c_{n}$ be the sequence constantly equal to $n$. Then $c_{n}(\boldsymbol{\alpha})=n$.

Proof. Recall that in Zermelo-Fraenkel set theory, natural numbers are coded as von Neumann naturals, i.e. 0 is the emptyset and, by induction, $n+1$ is the set $n \cup\{n\}$. Thus for $n=0$, the thesis follows from axiom 5 . Now proceed by induction and consider $c_{n+1}$. For all $m \in N, c_{n+1}(m)=n+1=n \cup\{n\}=$ $c_{n}(m) \cup\left\{c_{n}(m)\right\}$. By inductive hypothesis, $c_{n+1}(\boldsymbol{\alpha})=c_{n}(\boldsymbol{\alpha}) \cup\left\{c_{n}(\boldsymbol{\alpha})\right\}=$ $n \cup\{n\}=n+1$.

The next proposition shows that sequences that are eventually equal have

\footnotetext{
${ }^{5}$ Recall that, by definition, $\bigcup X=\{y: y \in x$ for some $x \in X\}$.
} 
the same ideal values, while sequences that are eventually different have different ideal values.

Proposition 3. Let $\varphi$ and $\psi$ be two given sequences.

(i) If $\varphi(m)=\psi(m)$ for all but a finite number of $m$, then $\varphi(\boldsymbol{\alpha})=\psi(\boldsymbol{\alpha})$.

(ii) If $\varphi(m) \neq \psi(m)$ for all but a finite number of $m$, then $\varphi(\boldsymbol{\alpha}) \neq \psi(\boldsymbol{\alpha})$.

Proof. (i). Let $\{m \in N: \varphi(m) \neq \psi(m)\}=\left\{n_{1}, \ldots, n_{k}\right\}$. Fix $n \in N$ with $\varphi(n)=\psi(n)$, and define the sequence $\zeta$ as follows. $\zeta(m)=n$ if $\varphi(m)=\psi(m)$, and $\zeta(m)=m$ otherwise. We claim that $\zeta(\boldsymbol{\alpha})=n$. Notice that for all $m \in N$, $\{\zeta(m)\} \backslash\{m\} \subseteq\{n\}$, hence $\{\zeta(\boldsymbol{\alpha})\} \backslash\{\boldsymbol{\alpha}\} \subseteq\{n\}$. Moreover, $\zeta(\boldsymbol{\alpha}) \neq \boldsymbol{\alpha}$ because $\zeta(m) \in\left\{n_{1}, \ldots, n_{k}, n\right\}$ for all $m \in N \Rightarrow \zeta(\boldsymbol{\alpha}) \in\left\{n_{1}, \ldots, n_{k}, n\right\}$, while $\boldsymbol{\alpha} \notin N$. Now, take $A=\operatorname{range}(\varphi) \cup \operatorname{range}(\psi)$ and define the sequence $\xi$ by setting $\xi(n)=\emptyset$ and $\xi(m)=A$ for $m \neq n$. Then $\{\varphi(m)\} \backslash\{\psi(m)\} \subseteq \xi(\zeta(m))$ for all $m \in N$ implies $\{\varphi(\boldsymbol{\alpha})\} \backslash\{\psi(\boldsymbol{\alpha})\} \subseteq(\xi \circ \zeta)(\boldsymbol{\alpha})=($ by axiom 7$)\left(\xi \circ c_{n}\right)(\boldsymbol{\alpha})=c_{\emptyset}(\boldsymbol{\alpha})=\emptyset$, and $\varphi(\boldsymbol{\alpha})=\psi(\boldsymbol{\alpha})$. (ii). Since $\{\varphi(m)\} \backslash\{\psi(m)\}=\{\varphi(m)\}$ for all but a finite number of $m$, by (i) it follows that $\{\varphi(\boldsymbol{\alpha})\} \backslash\{\psi(\boldsymbol{\alpha})\}=\{\varphi(\boldsymbol{\alpha})\}$, that is $\varphi(\boldsymbol{\alpha}) \neq \psi(\boldsymbol{\alpha})$. $\dashv$

We are now ready to define the nonstandard mapping $*$.

Definition. For any set $A$, let ${ }^{*} A$ be the ideal value of the sequence $c_{A}$ constantly equal to $A$, that is ${ }^{*} A=c_{A}(\boldsymbol{\alpha})$.

The map $*$ gives a 1-1 $\in$-homomorphism from the universal class of all sets into itself. Moreover, $*$ preserves all basic operations of sets. 6

Proposition 4. For every sets $A, B$, the following hold.

(i) $*\{A, B\}=\left\{{ }^{*} A,{ }^{*} B\right\}$.

(ii) $A \subseteq B \Leftrightarrow{ }^{*} A \subseteq{ }^{*} B$.

(iii) $A \in B \Leftrightarrow{ }^{*} A \in \overline{ }^{*} B$.

(iv) $A=B \Leftrightarrow{ }^{*} A={ }^{*} B$.

(v) $*(\bigcup A)=\bigcup\left({ }^{*} A\right)$.

(vi) $*(A \cup B)={ }^{*} A \cup{ }^{*} B$.

(vii) ${ }^{*}(A \cap B)={ }^{*} A \cap{ }^{*} B$.

(viii) * $(A \backslash B)=* A \backslash * B$.

(ix) ${ }^{*}\langle A, B\rangle=\left\langle{ }^{*} A,{ }^{*} B\right\rangle$.

$(x) *(A \times B)={ }^{*} A \times * B$.

(xi) ${ }^{*}\{\langle x, x\rangle: x \in A\}=\left\{\langle\xi, \xi\rangle: \xi \in{ }^{*} A\right\}$.

(xii) $*\{\langle x, y\rangle: x \in y \in A\}=\left\{\langle\xi, \eta\rangle: \xi \in \eta \in{ }^{*} A\right\}$.

(xiii) $*\{\langle x, y\rangle:\langle y, x\rangle \in A\}=\left\{\langle\xi, \eta\rangle:\langle\eta, \xi\rangle \in{ }^{*} A\right\}$.

$(x i v) *\{\langle x, y, z\rangle:\langle x, z, y\rangle \in A\}=\left\{\langle\xi, \eta, \zeta\rangle:\langle\xi, \zeta, \eta\rangle \in{ }^{*} A\right\}$.

$(x v) *\{x: \exists y\langle x, y\rangle \in A\}=\left\{\xi: \exists \eta\langle\xi, \eta\rangle \in{ }^{*} A\right\}$, i.e. ${ }^{*} \operatorname{dom}(A)=\operatorname{dom}\left({ }^{*} A\right)$.

(xvi) $*\{y: \exists x\langle x, y\rangle \in A\}=\left\{\eta: \exists \xi\langle\xi, \eta\rangle \in{ }^{*} A\right\}$, i.e. ${ }^{*} \operatorname{range}(A)=\operatorname{range}\left({ }^{*} A\right)$.

\footnotetext{
${ }^{6}$ Notice that the list given in proposition 4 includes all Gödel operations (see [JE] §11). As a consequence, the transfer principle could be directly proved (see [D2]). In this paper, the transfer principle will be proved by different means, through the representation theorem 8 .
} 
Proof. (i) Trivial by axiom 6. (ii), (v), (vi), (vii), (viii), (ix) and (x) directly follow from proposition 1. (iii) $A \in B \Leftrightarrow\{A\} \subseteq B \Leftrightarrow{ }^{*}\{A\}=\left\{{ }^{*} A\right\} \subseteq{ }^{*} B \Leftrightarrow$ ${ }^{*} A \in{ }^{*} B$. Also (iv) is a corollary of (ii). Property (xi) is straightforward. The remaining properties are all proved similarly one to the other. For instance, let us consider $(x v)$. If $\xi$ is a sequence with $\xi(n) \in \operatorname{dom}(A)$ for all $n \in N$, then there is a sequence $\eta$ with $\langle\xi(n), \eta(n)\rangle \in A$ for all $n \in N$. Hence $\langle\xi(\boldsymbol{\alpha}), \eta(\boldsymbol{\alpha})\rangle \in{ }^{*} A$, and $\xi(\boldsymbol{\alpha}) \in \operatorname{domain}\left({ }^{*} A\right)$. Vice versa, let $p \in \operatorname{domain}(A)$, i.e. $\langle p, q\rangle \in{ }^{*} A$ for some $q$. Then $\langle p, q\rangle=\zeta(\boldsymbol{\alpha})$ for some sequence $\zeta: N \rightarrow A$. Since $A \subseteq D \times R \Rightarrow^{*} A \subseteq$ ${ }^{*} D \times{ }^{*} R$ where $D=\operatorname{dom}(A)$ and $R=\operatorname{range}(A)$, there are sequences $\xi: N \rightarrow \bar{D}$ and $\eta: N \rightarrow R$ with $p=\xi(\boldsymbol{\alpha})$ and $q=\eta(\boldsymbol{\alpha})$. Notice that $\langle p, q\rangle=\nu(\boldsymbol{\alpha})$ where $\nu$ is the sequence $n \mapsto\langle\xi(n), \eta(n)\rangle$. Now, let $\pi: D \times R \rightarrow D$ be the projection on the first coordinate. $\xi(n)=(\pi \circ \nu)(n)$ for all $n \in N$ implies $p=\xi(\boldsymbol{\alpha})=(\pi \circ \nu)(\boldsymbol{\alpha})$. But $\nu(\boldsymbol{\alpha})=\langle p, q\rangle=\zeta(\boldsymbol{\alpha})$, thus by axiom $7, p=(\pi \circ \nu)(\boldsymbol{\alpha})=(\pi \circ \zeta)(\boldsymbol{\alpha})$ where $\pi \circ \zeta: N \rightarrow D$. We conclude that $p \in{ }^{*} D={ }^{*} \operatorname{dom}(A)$.

For every $A$, trivially $\left\{{ }^{*} a: a \in A\right\} \subseteq{ }^{*} A$. The following result gives a characterization of the fixed points of $*$.

Proposition 5. $\left\{{ }^{*} a: a \in A\right\}={ }^{*} A$ if and only if $A$ is finite.

Proof. If $A=\left\{a_{1}, \ldots, a_{k}\right\}$ is finite, we already saw that ${ }^{*} A=\left\{{ }^{*} a_{1}, \ldots,{ }^{*} a_{k}\right\}$. Vice versa, if $A$ is infinite there is a 1-1 mapping $f: N \rightarrow A$. Take a left inverse $g: A \rightarrow N$, i.e. a mapping with $g(f(n))=n$ for all $n \in N$. We claim that $f(\boldsymbol{\alpha}) \in{ }^{*} A$ and $f(\boldsymbol{\alpha}) \neq{ }^{*} a$ for every $a \in A$. Assume by contradiction that $f(\boldsymbol{\alpha})={ }^{*} a=c_{a}(\boldsymbol{\alpha})$ for some $a \in A$. Then, $\boldsymbol{\alpha}=1_{N}(\boldsymbol{\alpha})=(g \circ f)(\boldsymbol{\alpha})=$ $\left(g \circ c_{a}\right)(\boldsymbol{\alpha})=c_{g(a)}(\boldsymbol{\alpha})={ }^{*} g(a)$. This is impossible, because (by proposition 2) ${ }^{*} g(a)=g(a) \in N$ while $\boldsymbol{\alpha} \notin N$.

For each subset $A \subseteq N$, let $\chi_{A}: N \rightarrow\{0,1\}$ be its characteristic function. That is, $\chi_{A}(n)=1$ if $n \in A$ and $\chi_{A}(n)=0$ otherwise.

\section{Theorem 6.}

$\mathcal{U}=\left\{A \subseteq N: \chi_{A}(\boldsymbol{\alpha})=1\right\}$ is an ultrafilter over $N$ that contains no finite sets.

Proof. In the following, recall that 0 and 1 are identified with the emptyset $\emptyset$ and with the singleton $\{\emptyset\}$, respectively. Let $A$ be a given subset of $N$ and denote by $A^{c}$ its complement $N \backslash A$. For all $n \in N, \chi_{A}(n) \cup \chi_{A^{c}}(n)=\{\emptyset\}$ and $\chi_{A}(n) \cap \chi_{A^{c}}(n)=\emptyset$. Thus $\chi_{A}(\boldsymbol{\alpha}) \cup \chi_{A^{c}}(\boldsymbol{\alpha})=\{\emptyset\}$ and $\chi_{A}(\boldsymbol{\alpha}) \cap \chi_{A^{c}}(\boldsymbol{\alpha})=\emptyset$. Since every characteristic function takes its ideal value in the set $\{\emptyset,\{\emptyset\}\}$, the above equalities imply

- For every subset $A \subseteq N$,

$$
\text { (i) } \chi_{A}(\boldsymbol{\alpha})=1 \Rightarrow \chi_{A^{c}}(\boldsymbol{\alpha})=0 \text {; (ii) } \chi_{A}(\boldsymbol{\alpha})=0 \Rightarrow \chi_{A^{c}}(\boldsymbol{\alpha})=1 \text {. }
$$

Now, let us check the properties of filter. Assume $A, B \in \mathcal{U}$, i.e. $\chi_{A}(\boldsymbol{\alpha})=$ $\chi_{B}(\boldsymbol{\alpha})=1$. By property $(i), \chi_{A^{c}}(\boldsymbol{\alpha})=\chi_{B^{c}}(\boldsymbol{\alpha})=0$. Notice that $\chi_{\left(A^{c} \cup B^{c}\right)}(n)=$ $\chi_{A^{c}}(n) \cup \chi_{B^{c}}(n)$ for all $n \in N$, thus $\chi_{\left(A^{c} \cup B^{c}\right)}(\boldsymbol{\alpha})=\chi_{A^{c}}(\boldsymbol{\alpha}) \cup \chi_{B^{c}}(\boldsymbol{\alpha})=\emptyset \cup \emptyset=0$. Since $A \cap B=\left(A^{c} \cup B^{c}\right)^{c}$, by (ii) it follows that $\chi_{A \cap B}(\boldsymbol{\alpha})=1$, i.e. $A \cap B \in \mathcal{U}$. 
Now assume $A \supseteq B \in \mathcal{U}$. Then $\chi_{A}(n) \supseteq \chi_{B}(n)$ for all $n \in N \Rightarrow \chi_{A}(\boldsymbol{\alpha}) \supseteq$ $\chi_{B}(\boldsymbol{\alpha})=1$, hence $\chi_{A}(\boldsymbol{\alpha})=1$. The ultrafilter property is given by the above (i) and (ii). Finally, let $A$ be a finite subset of $N$. Then $\chi_{A}(n)=c_{\emptyset}(n)$ for all but a finite number of $n$, and by proposition $3, \chi_{A}(\boldsymbol{\alpha})=c_{\emptyset}(\boldsymbol{\alpha})=0$.

Proposition 7. Let $\varphi, \psi: N \rightarrow A$ be two sequences. Then

(i) $\varphi(\boldsymbol{\alpha})=\psi(\boldsymbol{\alpha}) \Leftrightarrow\{n \in N: \varphi(n)=\psi(n)\} \in \mathcal{U}$.

(ii) $\varphi(\boldsymbol{\alpha}) \in \psi(\boldsymbol{\alpha}) \Leftrightarrow\{n \in N: \varphi(n) \in \psi(n)\} \in \mathcal{U}$.

Proof. (i). Denote $\Lambda=\{n \in N: \varphi(n)=\psi(n)\}$. Assume first $\Lambda \in \mathcal{U}$. Let $\xi$ be the sequence such that $\xi(n)=A$ if $n \in \Lambda$ and $\xi(n)=0$ otherwise. Notice that $\xi=f \circ \chi_{\Lambda}$ where $f:\{0,1\} \rightarrow\{0, A\}$ is the function with $0 \mapsto 0$ and $1 \mapsto A . \chi_{\Lambda}(\boldsymbol{\alpha})=1=c_{1}(\boldsymbol{\alpha}) \Rightarrow \xi(\boldsymbol{\alpha})=\left(f \circ \chi_{\Lambda}\right)(\boldsymbol{\alpha})=\left(f \circ c_{1}\right)(\boldsymbol{\alpha})=c_{A}(\boldsymbol{\alpha})={ }^{*} A$. For all $n \in N,[\{\varphi(n)\} \backslash\{\psi(n)\}] \cap \xi(n)=0$, thus $[\{\varphi(\boldsymbol{\alpha})\} \backslash\{\psi(\boldsymbol{\alpha})\}] \cap \xi(\boldsymbol{\alpha})=$ 0 . But $\varphi(\boldsymbol{\alpha}) \in{ }^{*} A \Rightarrow\{\varphi(\boldsymbol{\alpha})\} \backslash\{\psi(\boldsymbol{\alpha})\}=0 \Rightarrow \varphi(\boldsymbol{\alpha})=\psi(\boldsymbol{\alpha})$. Vice versa, assume $\varphi(\boldsymbol{\alpha})=\psi(\boldsymbol{\alpha})$. For all $n \in N,[\{\varphi(n)\} \backslash\{\psi(n)\}] \cup \chi_{\Lambda}(n) \neq 0$, so $[\{\varphi(\boldsymbol{\alpha})\} \backslash\{\psi(\boldsymbol{\alpha})\}] \cup \chi_{\Lambda}(\boldsymbol{\alpha}) \neq 0$. Since $\{\varphi(\boldsymbol{\alpha})\} \backslash\{\psi(\boldsymbol{\alpha})\}=0$ by the hypothesis, we conclude $\chi_{\Lambda}(\boldsymbol{\alpha}) \neq 0$, i.e. $\Lambda \in \mathcal{U}$. (ii). Denote $\Gamma=\{n \in N: \varphi(n) \in \psi(n)\}$. If $\varphi(\boldsymbol{\alpha}) \in \psi(\boldsymbol{\alpha})$, then $\varphi(\boldsymbol{\alpha})=\xi(\boldsymbol{\alpha})$ with $\xi$ a sequence such that $\xi(n) \in \psi(n)$ for all $n \in N$. By (i), $\Lambda=\{n \in N: \varphi(n)=\xi(n)\} \in \mathcal{U}$, thus also $\Gamma \in \mathcal{U}$ because $\Gamma \supseteq \Lambda$. Vice versa, assume $\Gamma \in \mathcal{U}$. Let $\xi$ and $\zeta$ be the sequences where $\xi(n)=\{\varphi(n)\} \cap \psi(n)$ and $\zeta(n)=\{\varphi(n)\}$ for all $n \in N$. Notice that $\Lambda=\{n \in N: \xi(n)=\zeta(n)\} \in \mathcal{U}$ because $\Lambda \supseteq \Gamma \in \mathcal{U}$. Then, by (i), $\xi(\boldsymbol{\alpha})=\zeta(\boldsymbol{\alpha})$, i.e. $\{\varphi(\boldsymbol{\alpha})\} \cap \psi(\boldsymbol{\alpha})=\{\varphi(\boldsymbol{\alpha})\} \Leftrightarrow \varphi(\boldsymbol{\alpha}) \in \psi(\boldsymbol{\alpha})$.

Notice that $\mathcal{U}$ gives a nonatomic finitely additive two-valued measure $\mu$ : $\mathcal{P}(N) \rightarrow\{0,1\}$ defined for all subsets of $N$. Thus we could restate (i) in the above proposition as follows: "Two sequences have the same ideal value if and only if they are equal almost everywhere (with respect to $\mu$ )". Similarly (ii). For any set $A$, let $A^{N} / \mathcal{U}$ be the ultrapower of $A$ modulo the ultrafilter $\mathcal{U}$. Let $\pi_{A}$ be the function such that, for every sequence $\varphi: N \rightarrow A, \pi_{A}(\varphi(\boldsymbol{\alpha}))=[\varphi] \in A^{N} / \mathcal{U}$ is the $\mathcal{U}$-equivalence class of $\varphi$ modulo $\mathcal{U}$.

\section{Representation Theorem 8.}

For every set $A$, the function $\pi_{A}:{ }^{*} A \rightarrow A^{N} / \mathcal{U}$ is an $\in$-isomorphism and the following diagram commutes. ${ }^{7}$

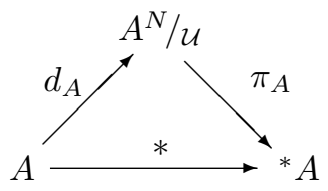

Moreover, for all $\in$-formulas $\sigma\left(x_{1}, \ldots, x_{n}\right)$ and elements $a_{1}, \ldots, a_{n} \in A$

$$
\langle A, \in\rangle \models \sigma\left(a_{1}, \ldots, a_{n}\right) \Leftrightarrow\left\langle{ }^{*} A, \in\right\rangle \models \sigma\left({ }^{*} a_{1}, \ldots,{ }^{*} a_{n}\right)
$$

\footnotetext{
${ }^{7} d_{A}$ denotes the diagonal embedding of $A$ into its ultrapower.
} 
Proof. By the previous proposition, $\varphi(\boldsymbol{\alpha})=\psi(\boldsymbol{\alpha}) \Leftrightarrow\{n \in N: \varphi(n)=\psi(n)\} \in$ $\mathcal{U} \Leftrightarrow[\varphi]=[\psi]$. Thus $\pi_{A}$ is $1-1$, and also it is trivially onto. Besides, $\varphi(\boldsymbol{\alpha}) \in$ $\psi(\boldsymbol{\alpha}) \Leftrightarrow\{n \in N: \varphi(n) \in \psi(n)\} \in \mathcal{U} \Leftrightarrow A^{N} / \mathcal{U} \models[\varphi] \in[\psi]$ and $\pi_{A}$ is an $\in$-homomorphism. The commutativity of the diagram is immediate. In fact, for every $a \in A, \pi_{A}\left({ }^{*} a\right)=\pi\left(c_{a}(\boldsymbol{\alpha})\right)=\left[c_{a}\right]=d_{A}(a)$, where $c_{a}$ is the sequence constantly equal to $a$. Finally, the equivalence for $\in$-formulas $\sigma$ is given by $\operatorname{Los}$ theorem on ultrapowers. ${ }^{8} \quad \dashv$

We are now ready to prove the two fundamental principles of nonstandard analysis, namely the transfer principle and the saturation property. As in the superstructure approach, by definition we say that a set $x$ is internal if $x \in$ ${ }^{*} A$ for some $A$. Equivalently, $x$ is internal if $x=\varphi(\boldsymbol{\alpha})$ is the ideal value of some sequence $\varphi$. Notice that the class $I$ of internal elements is transitive, i.e. elements of internal sets are internal.

Theorem 9. Countable Saturation.

Let $\mathcal{F}$ be a countable family of internal sets. If every finite subfamily of $\mathcal{F}$ has nonempty intersection, then $\mathcal{F}$ has nonempty intersection.

Proof. Let $\mathcal{F}=\left\{F_{n}: n \in N\right\}$ and take $A$ with $F_{n} \in{ }^{*} A$ for all $n$. For each $n \in N$, choose $a_{n} \in \bigcap_{i=0}^{n} F_{i}$, and consider the function $\varphi: N \rightarrow A$ where $n \mapsto a_{n}$. For each $k \in N,\left\{n \in N: \varphi(n) \in F_{k}\right\}=\{n \in N: n \geq k\}$ is a cofinite set, hence an element of $\mathcal{U}$. By the Representation theorem, $A^{N} / \mathcal{U}=[\varphi] \in d_{A}\left(F_{k}\right) \Leftrightarrow$ $\left\langle{ }^{*} A, \in\right\rangle \models \pi_{A}^{-1}([\varphi]) \in F_{k}$. Since this holds for each $k \in N$, we have found an element $a=\pi_{A}^{-1}([\varphi]) \in \bigcap \mathcal{F}$.

Theorem 10. Transfer Principle.

For every bounded $\in$-formula $\sigma\left(x_{1}, \ldots, x_{n}\right)$ and elements $a_{1}, \ldots, a_{n}:{ }^{9}$

$$
\sigma\left(a_{1}, \ldots, a_{n}\right) \Leftrightarrow \sigma^{I}\left({ }^{*} a_{1}, \ldots,{ }^{*} a_{n}\right)
$$

Proof. Take $A$ a transitive set containing $a_{1}, \ldots, a_{n}$ and consider the $\in$ isomorphism $\pi_{A}:{ }^{*} A \rightarrow A^{N} / \mathcal{U}$ and the diagonal embedding $d_{A}: A \rightarrow A^{N} / \mathcal{U}$ as given by the Representation theorem. It is easily seen that also ${ }^{*} A$ is transitive. Since bounded formulas are absolute between transitive classes ${ }^{10}$, then $\sigma\left(a_{1}, \ldots, a_{n}\right) \Leftrightarrow\langle A, \in\rangle \models \sigma\left(a_{1}, \ldots, a_{n}\right) \Leftrightarrow\left\langle{ }^{*} A, \in\right\rangle \models \sigma\left({ }^{*} a_{1}, \ldots, a_{n}\right) \Leftrightarrow$ $\sigma^{I}\left({ }^{*} a_{1}, \ldots,{ }^{*} a_{n}\right) . \quad \dashv$

Finally, the next theorem gives a foundational justification to ZFC $[\boldsymbol{\alpha}]$.

\section{Theorem 11.}

$Z F C[\boldsymbol{\alpha}]$ is equiconsistent with $Z F C$.

\footnotetext{
${ }^{8}$ See $[\mathrm{CK}] \S 4.1$.

${ }^{9}$ Recall that a formula $\sigma$ is bounded if every quantifier occurs in bounded form $\forall x \in I$ (i.e. $\forall x x \in I \rightarrow \ldots$ ) or $\exists x \in I$ (i.e. $\exists x x \in I \wedge \cdots$ ). By $\sigma^{I}$ we denote the $I$-relativization of $\sigma$, that is the formula obtained from $\sigma$ by replacing each quantifier $\forall x$ and $\exists x$ with its restricted form $\forall x \in I$ (i.e. $\forall x x \in I \rightarrow \ldots$ ) and $\exists x \in I$ (i.e. $\exists x x \in I \wedge \cdots$ ), respectively.

${ }^{10}$ See $[\mathrm{JE}] \S 14$.
} 
Proof. If $M$ is a model of ZFC $[\boldsymbol{\alpha}]$, then its wellfounded part $\mathbf{W} \mathbf{F}^{M}=\{m \in$ $M: M \models$ " $m$ is wellfounded" $\}$ is a model of ZFC. Vice versa, let us assume the consistency of ZFC. We need to find a model of ZFC $[\boldsymbol{\alpha}]$. In the following, we will work in ZFBC, an equiconsistent variant of Zermelo-Fraenkel set theory where a bijection $\mathbf{C}: \mathbf{O N} \rightarrow \mathbf{V}$ between the class of ordinals and the universal class of all sets is available (thanks to a new binary relation $C$ considered in the language), and where regularity is replaced by Boffa's superuniversality axiom. Superuniversality is a strong anti-foundation axiom which implies the existence of a transitive collapse for every (not necessarily wellfounded) extensional structure. ${ }^{11}$ The construction below closely follows arguments in the proof of the Extension Principle of $[\mathrm{BH}]$. Thus we give here only a sketch and refer the reader to that paper for details. 12 The universal class $\mathbf{V}$ is an increasing union of transitive sets indexed by ordinals. For instance, $\mathbf{V}=\bigcup\left\{W_{\alpha}: \alpha \in \mathbf{O N}\right\}$ where $W_{\alpha}$ is the transitive closure of the set $\{\mathbf{C}(\beta): \beta<\alpha\}$. Now fix a nontrivial ultrafilter $D$ over $N$. As a consequence of superuniversality, one gets transitive collapses $\pi_{\alpha}: W_{\alpha}{ }^{N} / D \rightarrow T_{\alpha}$ such that for all $\alpha \leq \beta, \pi_{\alpha}$ is the restriction of $\pi_{\beta}$ to $W_{\alpha}{ }^{N} / D$ :

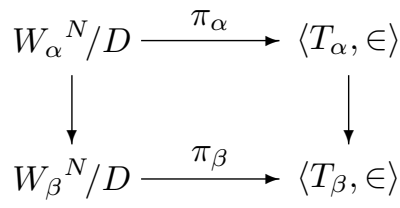

The class $\boldsymbol{\pi} \doteq \bigcup\left\{\pi_{\alpha}: \alpha \in \mathbf{O N}\right\}$ is a transitive collapse for the $D$-ultrapower of the universe $\mathbf{V}^{N} / D=\{[f]: f$ is a function with $\operatorname{dom}(f)=N\}$. We remark that in order to make each equivalence class $[f]$ a set (and not a proper class) some caution is needed. In ZFC, this problem is overcome by Scott's trick. In our nonwellfounded context (where a rank is not available) one can define $[f]$ as the least function in the well-ordering of the universe (given by $\mathbf{C}$ ) which is $D$-equivalent to $f$. Doing so, the collection $\{[f]: f$ is a function with $\operatorname{dom}(f)=N\}$ is actually the extension of a formula in ZFBC. Now, let $\boldsymbol{\pi}\left(\left[1_{N}\right]\right)$ be the interpretation of the constant symbol $\boldsymbol{\alpha}$, where $1_{N}: N \rightarrow N$ denotes the identity map on $N$. For all $N$-sequences $f$, let us interpret $f(\boldsymbol{\alpha})=\boldsymbol{\pi}([f])$. Notice that the nonstandard embedding * corresponds to the bounded elementary embedding $\left(\bigcup_{\alpha \in O N} \pi_{\alpha} \circ d_{\alpha}\right): \mathbf{V} \rightarrow \mathbf{V}$, where $d_{\alpha}: W_{\alpha} \rightarrow W_{\alpha}{ }^{N} / D$ are the diagonal embeddings. All axioms of $\mathrm{ZFC}[\boldsymbol{\alpha}]$ directly follow from properties of the ultrapower construction.

\footnotetext{
${ }^{11} \mathrm{ZFBC}$ was first proposed as a foundational framework for the practice of nonstandard methods by D. Ballard and K. Hrbáček in [BH] (see that paper for a precise formulation). That theory was also adopted by the author for the construction of superstructure-like nonstandard universes in [D1]. A proof of the consistency of superuniversality is in [Bo].

${ }^{12}$ See also $[\mathrm{BD}]$, where a stronger theory than $\mathrm{ZFC}[\boldsymbol{\alpha}]$ is given a faithful interpretation in $\mathrm{ZFC}$
} 


\section{References}

[Be] V. Benci, A Construction of a Nonstandard Universe, in Advances in Dynamical Systems and Quantum Physics, S. Albeverio et al., eds., World Scientific, 1995, pp. 207-237.

[BD] V. Benci And M. Di NAsso, Expanding ZFC for the Practice of Nonstandard Analysis, in preparation.

[BH] D. Ballard And K. HrbÁC̆eK, Standard Foundations for Nonstandard Analysis, J. Symb. Logic, vol. 57 (1992), pp. 471-478.

[Bo] M. BoffA, Forcing et Négation de l'Axiome de Fondement, Mém. Acad. Sc. Belg., tome XL, fasc. 7, 1972.

[CK] C.C. Chang And H.J. Keisler, Model Theory, 3rd edition, NorthHolland, 1990.

[D1] M. Di Nasso, Pseudo-Superstructures as Nonstandard Universes, J. Symb. Logic, vol. 63 (1998), pp. 222-236.

[D2] M. Di NAsso, Some Remarks on Nonstandard Set Theory, in preparation.

[JE] JECH, Set Theory, Academic Press, 1978.

[LA] D. LAugwitz, $\Omega$-Calculus as a Generalization of Field Extension An Alternative Approach to Nonstandard Analysis, in Nonstandard Analysis - Recent Developments (Proceedings Victoria Conference 1980), A. Dold and B. Eckmann, eds., Lecture Notes in Mathematics 983, Springer-Verlag, 1983, pp. 120-133.

[SL] C. Schmieden And D. Laugwitz, Eine Erweiterung der Infinitesimalrechnung, Math. Zeitschr., vol. 69 (1958), pp. 1-39.

Dipartimento di Matematica Applicata

UNIVERSITÁ Di PISA

VIA BonanNo 25/B

56126 Pisa, ITALY

EMAIL: dinasso@dma.unipi.it 